\title{
Simulation of Particle Behavior for Different Hoppers
}

\author{
Panpan Zhang1, 2, a, Gang Chen ${ }^{3, \mathrm{~b}}$ and Jiahai Zhou ${ }^{1, c, ~ *}$ \\ ${ }^{1}$ China Waterborne Transport Research Institute, Beijingm100088, China. \\ ${ }^{2}$ Key Laboratory of Logistics Equipment \& Control Engineering, Beijing 100088, China. \\ ${ }^{3}$ Rizhao Port Group Co., Ltd, Rizhao 276826, China. \\ azhangpanpan@wti.ac.cn, ${ }^{b}$ rzchgang@126.com, ${ }^{\mathrm{c}}$ zhoujh@wti.ac.cn.
}

Keywords: port, hopper, bulk cargo, DEM, particle behavior.

\begin{abstract}
With development of logistics and ports, port throughput of bulk cargo has been increasing. In the port, the bulk cargo conveying system plays a very important part. The transfer station is critical for bulk cargo distributing and the hopper is an important part in the transfer station. In this paper, different hoppers were designed. A discrete element method (DEM) software package EDEM was introduced to evaluate the particle behavior for different hoppers. The hopper angle has great influence on the dust particle behavior.
\end{abstract}

\section{Introduction}

As the economy booms all around the world, the throughput of bulk cargo has been increasing. In the bulk cargo conveying system, the hopper in the transfer station is of great important because the hopper is usually used for truck or train loading. Besides, the air pollution caused by the hopper loading is a major problem shown in Figure 1. In recent years, researchers focus on the study of particle behavior and dust suppression [1-4].

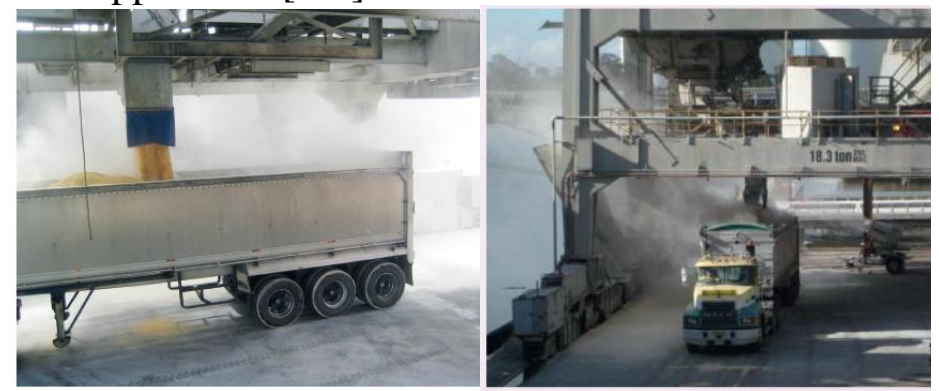

Figure 1. the air pollution caused by the hopper loading.

The discrete element method is popularly used for particle behavior evaluation. The software package EDEM was developed based on DEM and can provide detailed analysis of bulk cargo conveying process shown in Figure 2. The software package can help to evaluate the particle behavior of bulk cargo.
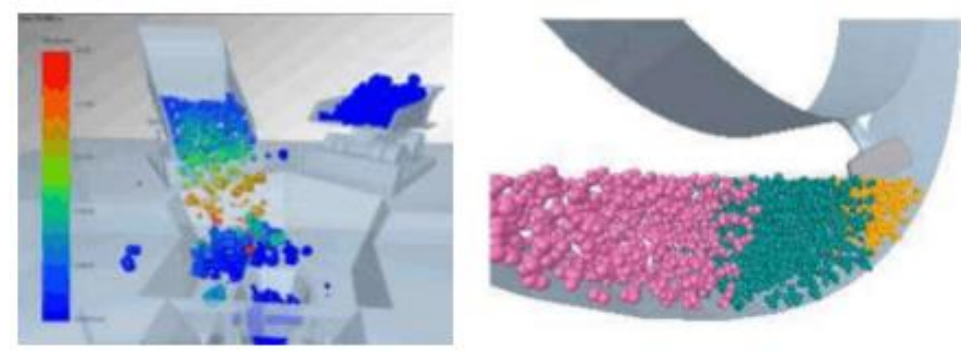

Figure 2. the interface of the software EDEM.

The hopper in the transfer station is a key element in the bulk cargo handling system. And the performance of the hopper has influence to the cargo handling. In this paper, different hoppers were designed. A DEM software package was chosen and simulations was carried out to evaluate the particle behaviors of different hoppers. 


\section{Simulation of Different Hoppers}

\subsection{Hopper Design.}

To investigate the particle behavior of different hoppers, different hoppers shown in Figure 3 were designed.

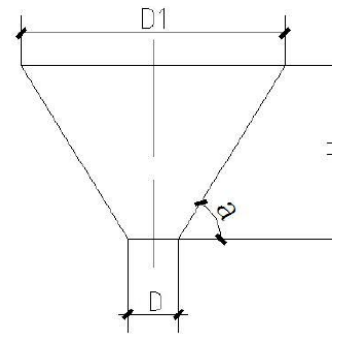

Figure 3. the structure of the hopper.

In Figure 3, the diameter of the inlet marked as D1 and the diameter of the outlet marked D2 were set as the fixed value. The angle of the hopper $\alpha$ is a viable. The parameters of hoppers were shown in Table 1.

Table 1. parameters of different hoppers.

\begin{tabular}{cccc}
\hline Item & $\mathrm{D} 1(\mathrm{~mm})$ & $\mathrm{D}(\mathrm{mm})$ & $\alpha\left(^{\circ}\right)$ \\
\hline 1 & 190 & 30 & 52 \\
2 & 190 & 30 & 54 \\
3 & 190 & 30 & 56 \\
4 & 190 & 30 & 58 \\
5 & 190 & 30 & 60 \\
6 & 190 & 30 & 62 \\
\hline
\end{tabular}

\subsection{Simulation Setting}

In simulation, corn was choosing as the material and $1 \%$ dust was contained in the corn. The physical parameters of the corn and the dust were shown in Table2. The equivalent particle diameter of corn is $4.5 \mathrm{~mm}$ and the equivalent particle diameter of dust is $0.4 \mathrm{~mm}$. During the corn unloading simulation process, the mass flow rate of corn is $0.07 \mathrm{~kg} / \mathrm{s}$ and the mass flow rate of dust is 0.0007 $\mathrm{kg} / \mathrm{s}$.

Table 2. physical parameters of the corn and the dust.

\begin{tabular}{cccc}
\hline Item & Density $/ \mathrm{kg} \cdot \mathrm{m}^{-3}$ & shear modulus $/ \mathrm{Pa}$ & Poisson's ratio \\
\hline Corn & 800 & $1.37 \mathrm{e}+08$ & 0.4 \\
Dust & 2000 & $1 \mathrm{e}+08$ & 0.25 \\
\hline
\end{tabular}

\section{Results and Discussion}

Figure 4 reveals the dust distribution of different hoppers. It is shown that as the angle increases, the material flow width will show a decreasing trend.
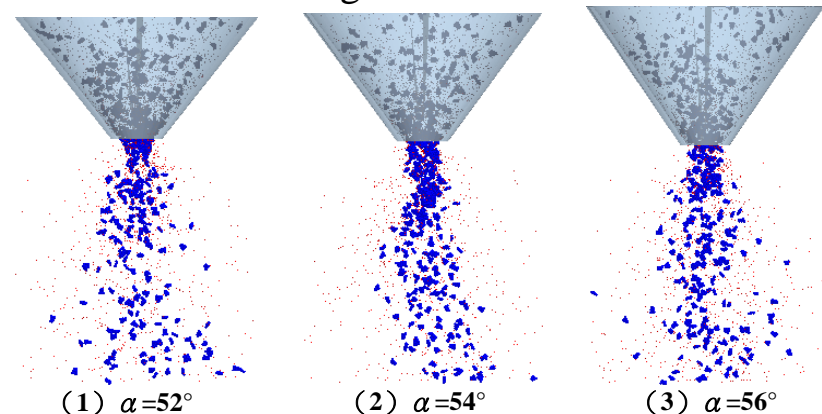

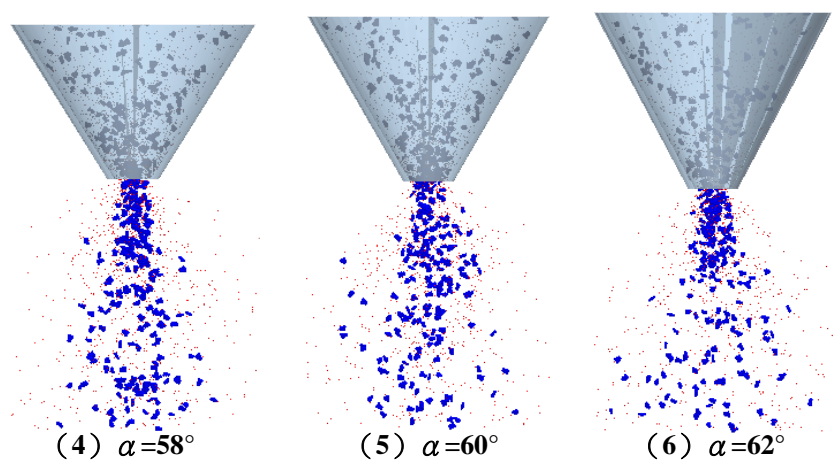

Figure 4. the distribution diagram of corn and dust.

In order to evaluate the distribution degree, 20 different concentric circles were chosen around the hopper outlet. The diameters for the concentric circles are $10 \mathrm{~mm}, 20 \mathrm{~mm}, 30 \mathrm{~mm}, 40 \mathrm{~mm} .200 \mathrm{~mm}$ shown in figure 5 . And then the number of dust particles for each circle was calculated and the curve for the dust distribution shown in Fig. 6 can be drawn.
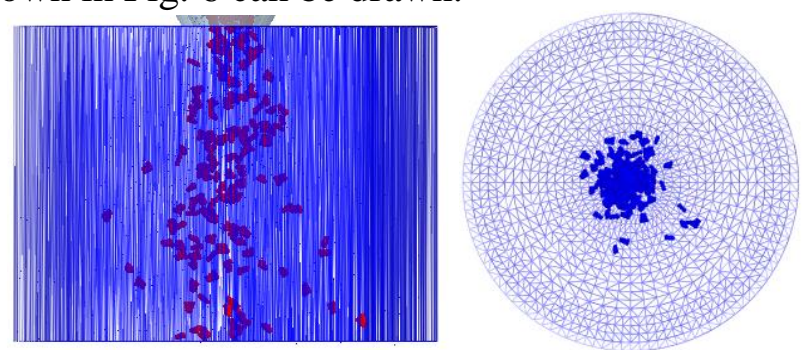

Figure 5. the concentric circles of the analysis zone.

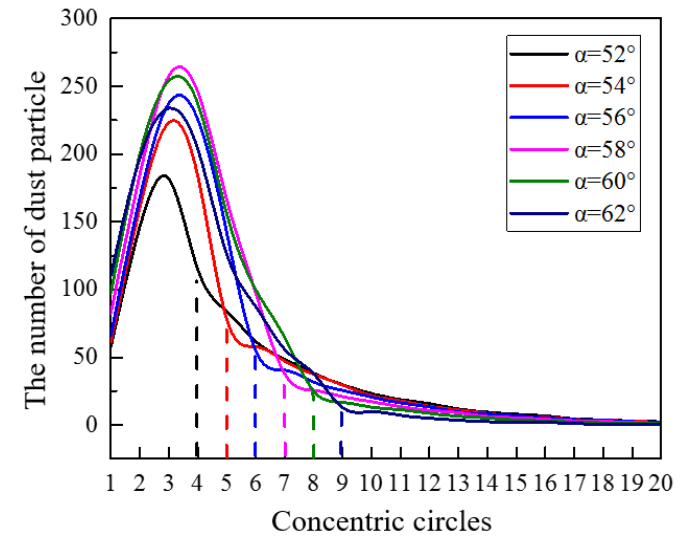

Figure 6. the number of dust particle for different hoppers.

From the chart, it can be concluded that the material flow is more concentrated with the increase of the hopper inclination angle, which contributes to the dust suppression. In order to investigate the dust contribution, the percentage of dust particle was introduced. Figure 7 reveals that the percentage of dust particle. It shows a similar trend to the material flow. The smaller the hopper angle is, the smaller the percentage of dust particles in the central area is and the greater the percentage of dust particles in the edge area is. And the material flow is more dispersed. On the contrary, the bigger the hopper angle is, the bigger the percentage of dust particles in the central area is and the smaller the percentage of dust particles in the edge area is. The material flow is more concentrated. 


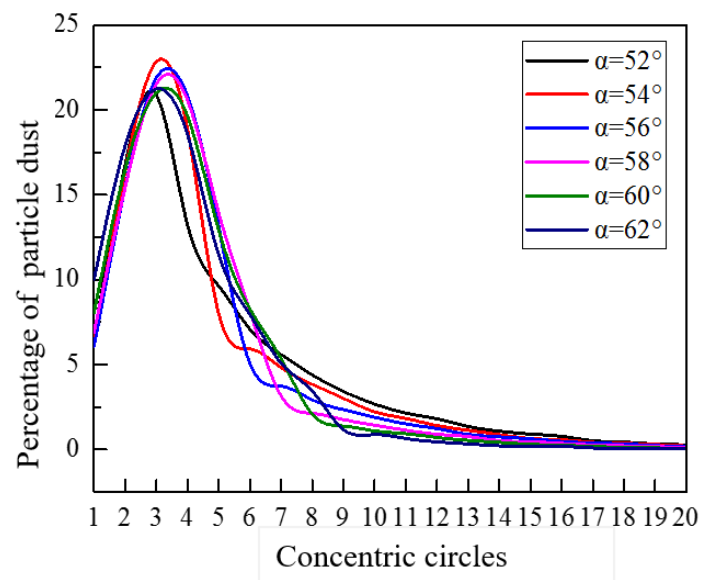

Figure 7. the percentage of dust particle for different hoppers.

\section{Summary}

In this paper, the particle behaviors of different hoppers were analyzed via the DEM software package EDEM. The results show that the structure of the hopper has direct influence of the particle behavior. With the angle of the hopper increasing, the flow of corn was more concentrated. The study will provide help for the hopper design.

\section{References}

[1]. Wypych P., Cook D., Cooper P. Controlling dust emissions and explosion hazards in powder handling plants [J]. Chemical Engineering and processing.2005, 44:323-326.

[2]. Liu Z.Q., Ma L.Y., Zhao X. Y. Experimental Study the affection of the Dust Emission on Sand Stockpile by the Height of Windbreak Wall and Wind Speed. Applied Mechanics and Materials, 2014, 823:989-994.

[3]. Tan C, Zhang K.P., Research advance in granular flow mathematical model, Journal of Hebei University of Science and Technology, 2013, 34 (4): 293-296.

[4]. Sommerfeld M. Particle dispersion in turbulent flow: the effect of particle size distribution. Particle \& particle systems characterization, 1990, 7:209-220. 